\title{
Axitinib versus sorafenib as a second-line therapy in Asian patients with metastatic renal cell carcinoma: results from a randomized registrational study
}

\author{
This article was published in the following Dove Press journal: \\ OncoTargets and Therapy \\ 8 June 2015 \\ Number of times this article has been viewed
}

\author{
Shukui Qin,' Feng $\mathrm{Bi}^{2}{ }^{2}$ Jie \\ Jin, ${ }^{3}$ Ying Cheng, ${ }^{4}$ Jun Guo, ${ }^{5}$ \\ Xiubao Ren, ${ }^{6}$ Yiran Huang, ${ }^{7}$ \\ Jamal Tarazi, ${ }^{8}$ Jie Tang, \\ Connie Chen, ${ }^{9}$ Sinil Kim, ${ }^{8}$ \\ Dingwei $\mathrm{Ye}^{10}$
}

'Department of Medical Oncology, PLA Cancer Center, Nanjing Bayi Hospital, Nanjing, Jiangsu, People's Republic of China; ${ }^{2}$ Department of Medical Oncology, West China Hospital of Sichuan University, Chengdu, Sichuan Province, People's Republic of China; ${ }^{3}$ Department of Urology, Peking University First Hospital, Beijing, People's Republic of China; ${ }^{4}$ Department of Oncology, Jilin Provincial Cancer Hospital, Changchun, Jilin Province, People's Republic of China; ${ }^{5}$ Department of Renal Cancer and Melanoma, Peking University Cancer Hospital/ Institute, Beijing, People's Republic of China; ${ }^{6}$ Department of Biology Treatment, Tianjin Oncology Hospital, Tianjin, People's Republic of China; ${ }^{7}$ Department of Urology, Renji Hospital, Shanghai Jiao Tong University School of Medicine, Shanghai, People's Republic of China; ${ }^{8}$ Clinical Development, Pfizer Oncology, San Diego, CA, USA; ' $\mathrm{Global}$ Outcomes Research, Pfizer Inc., New York, NY, USA; ${ }^{10}$ Department of Urology, Fudan University Shanghai Cancer Center, Shanghai, People's Republic of China

Correspondence: Dingwei Ye Department of Urology, Fudan University Shanghai Cancer Center, 270 Dong An Road, Shanghai 200032, People's Republic of China

Tel $+861370166357 \mid$

Fax +862164438640

Email dwyeli@163.com
Background: This registrational trial evaluated the efficacy, safety, and patient-reported outcomes of axitinib versus sorafenib as a second-line treatment in Asian patients with clearcell metastatic renal cell carcinoma (mRCC).

Methods: In this open-label, multicenter study, previously treated Asian patients with clearcell mRCC were stratified by Eastern Cooperative Oncology Group performance status and prior therapy and randomized in a 2:1 ratio to receive axitinib (5 mg twice daily) or sorafenib (400 mg twice daily). The primary end point was progression-free survival (PFS) assessed by a masked independent review committee.

Results: A total of 204 Asian patients received axitinib ( $n=135)$ or sorafenib $(n=69)$. Median PFS (95\% confidence interval [CI]) was 6.5 (4.7-9.1) months with axitinib versus 4.8 (3.0-6.5) months with sorafenib (hazard ratio, $0.731 ; 95 \% \mathrm{CI}, 0.506-1.058$; one-sided $P=0.0531$ ). The objective response rate $(95 \% \mathrm{CI})$ was $23.7 \%(16.8 \%-31.8 \%)$ with axitinib versus $10.1 \%(4.2 \%-19.8 \%)$ with sorafenib. Common, grade $\geq 3$, all-causality adverse events were hypertension (19.3\%), weight decrease (5.2\%), and proteinuria (5.2\%) with axitinib and hypertension $(8.7 \%)$ and palmar-plantar erythrodysesthesia (7.2\%) with sorafenib. In a time-to-deterioration composite end point of death, progression, and worsening of Functional Assessment of Cancer Therapy Kidney Symptom Index score, patients treated with axitinib demonstrated a $17 \%-24 \%$ risk reduction compared with sorafenib-treated patients.

Conclusion: Axitinib is clinically active and well tolerated in previously treated Asian patients with mRCC, consistent with the results from the global Phase III trial. These results establish axitinib as a second-line treatment option for Asian patients with $\mathrm{mRCC}$.

Keywords: axitinib, renal cell carcinoma, sorafenib, vascular endothelial growth factor receptor inhibitor

\section{Introduction}

In 2012, kidney cancer was diagnosed in more than 66,000 people in China and resulted in more than 25,000 deaths, a twofold increase in the number of deaths from 2008. ${ }^{1,2}$ Renal cell carcinoma (RCC) accounts for $\sim 90 \%$ of kidney cancers, of which clear-cell carcinoma is the predominant histological subtype, accounting for $85 \%$ of RCC. ${ }^{3}$

In many countries, the management of metastatic RCC (mRCC) has changed dramatically with the introduction of molecularly targeted agents such as sunitinib, ${ }^{4-7}$ pazopanib, ${ }^{8-10}$ sorafenib, ${ }^{11-14}$ temsirolimus, ${ }^{15}$ and everolimus. ${ }^{16}$ However, according to the resource-stratified guidelines developed at the 2012 Asian Oncology Summit, ${ }^{17}$ interferon $\alpha$ is still commonly used in Japan and considered the most cost-effective 
treatment in China. ${ }^{18}$ The availability of Asian-specific safety and efficacy data from a randomized clinical trial comparing one targeted agent versus another is obviously an important factor for making the decision to use a targeted agent in Asian populations. However, most of the available Asian-specific data come from single-arm Phase II, expanded access, or retrospective clinical studies. ${ }^{4-7,11-16,19}$

Axitinib, a potent and selective second-generation inhibitor of vascular endothelial growth factor receptors (VEGFRs) 1,2 , and $3,{ }^{20}$ is approved in the United States (Inlyta ${ }^{\circledR}$; Pfizer Inc, New York, NY, USA ${ }^{21}$ ), European Union, Japan, Korea, and other countries for the treatment of advanced RCC after failure of prior systemic therapy. In the global Phase III AXIS trial, axitinib improved progression-free survival (PFS) compared with sorafenib in patients with mRCC $(\mathrm{N}=723)$ after failure of one prior systemic therapy. ${ }^{22}$ Median PFS was 6.7 months with axitinib versus 4.7 months with sorafenib (hazard ratio [HR], 0.665; 95\% confidence interval [CI], $0.544-0.812$; one-sided $P<0.0001$ ). In a subgroup analysis of patients from Japan enrolled in AXIS, ${ }^{23}$ axitinib resulted in longer PFS and higher objective response rate (ORR) compared with sorafenib, consistent with the results obtained in the overall population. ${ }^{22}$ The nature and incidence of adverse events (AEs) observed in Japanese patients were generally similar to those reported in the overall population; however, AEs more frequently reported by Japanese patients treated with axitinib included hypertension and hypothyroidism.

Furthermore, treatment with axitinib had a statistically significant advantage compared with sorafenib on the composite end point of time-to-treatment deterioration, defined as death, disease progression, or worsening of symptoms (based on the Functional Assessment of Cancer Therapy Kidney Symptom Index [FKSI] questionnaire and FKSIDisease-Related Symptoms [FKSI-DRS]). ${ }^{22}$ The time-todeterioration FKSI-15 composite end point showed a $17 \%$ reduction in risk for axitinib versus sorafenib (HR, 0.829; 95\% CI, 0.701-0.981; one-sided $P=0.014$ ) and the time-todeterioration FKSI-DRS composite end point showed a $16 \%$ risk reduction for axitinib versus sorafenib (HR, 0.838; 95\% CI, 0.707-0.993; one-sided $P=0.0203)$. $^{22}$

An ongoing study in previously treated Asian patients with advanced RCC was designed to support the registration of axitinib in China and to satisfy regulatory requirements in that country. As such, the study has a similar design to the previous Phase III AXIS trial in which patients with advanced RCC were treated with axitinib following failure of one prior systemic first-line regimen. ${ }^{22}$ Based on Phase I studies that showed comparable axitinib pharmacokinetics between white and Japanese $\mathrm{e}^{24,25}$ or Chinese ${ }^{26}$ populations, axitinib dosage used in this Asian population was similar to that used in the predominantly white population in the global AXIS trial. Here, we report the results from this randomized registrational trial that was designed to prospectively evaluate the efficacy, safety, and patient-reported outcomes of axitinib versus sorafenib in Asian patients with mRCC after failure of first-line systemic therapy.

\section{Methods}

\section{Patients}

Key eligibility criteria for participation in this study included males or nonpregnant females aged 18 years or older; histologically or cytologically confirmed mRCC with a clearcell component; unidimensionally measurable disease by Response Evaluation Criteria in Solid Tumors (RECIST, version 1.0); ${ }^{27}$ progressive disease per RECIST after one prior first-line sunitinib- or cytokine-containing regimen for metastatic disease; adequate renal, hepatic, and hematologic function; Eastern Cooperative Oncology Group performance status (ECOG PS) 0 or 1 ; $\geq 2$ weeks since prior systematic treatment, radiotherapy, or surgery; no uncontrolled hypertension (defined as blood pressure $>140 / 90 \mathrm{mmHg}$ ); and life expectancy $\geq 12$ weeks. Antihypertensive therapy was permitted, as well as prior adjuvant interferon and/or interleukin therapy, if recurrence occurred $>6$ months after last dose.

Key exclusion criteria included current use or anticipated need for strong inhibitors of cytochrome P450 (CYP) 3A4/5 or inducers of CYP3A4/5 or CYP1A2; anticoagulant therapy with oral vitamin $\mathrm{K}$ antagonists; active seizure disorder or evidence of brain metastases, spinal cord compression, or carcinomatous meningitis; myocardial infarction, uncontrolled angina, coronary or peripheral artery bypass graft, symptomatic congestive heart failure, cerebrovascular accident, or transient ischemic attack within prior 12 months; deep vein thrombosis or pulmonary embolism within prior 6 months; known HIV or AIDS-related disease; or history of malignancy other than RCC.

\section{Study design}

This ongoing, multicenter, open-label, randomized registrational trial was originally designed to evaluate axitinib or sorafenib in previously treated Asian patients with mRCC. The study was conducted at 30 centers in seven countries: China (20 centers), India (one center), Malaysia (one center), the Philippines (four centers), Taiwan (two centers), Ukraine (one center), and the United States (one center). In 2009, the trial was amended to include a global 
treatment-naïve population. The amended study was designed to analyze this population separately from the second-line population; results are reported elsewhere. ${ }^{28} \mathrm{We}$ report results from the previously treated Asian population.

This study was conducted in compliance with the Declaration of Helsinki and International Conference on Harmonization Good Clinical Practice Guidelines; all local regulatory requirements were followed and, in particular, those affording greater protection to the safety of trial participants. The final protocol, amendments, and informed consent documents were reviewed and approved by an institutional review board or independent ethics committee at each study center. All patients gave written informed consent before entering the study. This trial is registered on ClinicalTrials. gov, identifier NCT00920816.

\section{Randomization and masking}

Patients were randomly assigned in a 2:1 ratio, using a centralized registration system, to receive either axitinib at a starting dose of $5 \mathrm{mg}$ twice daily (BID) or sorafenib $400 \mathrm{mg}$ BID. Patients were stratified by ECOG PS ( 0 or 1$)$ and by prior therapy (sunitinib- or cytokine-containing).

All planned efficacy analyses were performed using stratification data collected from the interactive voice response system. Randomization lists were generated using permuted blocks with a block size of six (four axitinib to two sorafenib) within each ECOG PS stratum and prior therapy stratum. The randomization list was generated by the central randomization administrator, and the treatment groups were obtained by the investigating site via a web interface. Patients and investigators were unmasked to treatment allocation. The radiologists who participated in the independent review committee (IRC) assessment of PFS (the primary end point) were masked to treatment allocation.

\section{Procedures}

Study treatments were administered in cycles of 4 weeks' duration. Axitinib was taken orally with food at a starting dose of $5 \mathrm{mg}$ BID and sorafenib was taken orally without food ( $\geq 1$ hour before or 2 hours after eating) at a starting dose of $400 \mathrm{mg}$ BID. Axitinib dose increases, as well as axitinib and sorafenib dose reductions and interruptions, were based on tolerability, as previously described..$^{22,29}$

\section{Assessments}

Baseline tumor assessments included computed tomography (CT)/magnetic resonance imaging (MRI) scans of the brain, chest, abdomen, and pelvis and a bone scan. CT/MRI (not including brain) and bone scan (only if lesions at baseline) were repeated at week 6 , week 12 , every 8 weeks thereafter, and at the end of study treatment. All radiographic images and bone scans were centrally reviewed by an IRC masked to treatment allocation.

All observed or patient-reported AEs (serious and nonserious), the severity of the events, and the investigator's opinion of the relationship to the study treatment were documented throughout the study period using the Common Terminology Criteria for Adverse Events version 3.0. Physical examinations were conducted at baseline, day 1 (if $>7$ days since baseline), week 2 , week 4 , every 4 weeks thereafter, and at the end of study treatment. Blood pressure was monitored at each clinic visit and BID at home by patients throughout the study period. Assessment of ECOG PS was conducted at baseline, day 1, every 4 weeks, and at the end of study treatment. Laboratory tests for hematology and blood chemistry were conducted at baseline, day 1 (if $>7$ days since baseline), every 4 weeks, and at the end of study treatment. Urinalysis was conducted every 4 weeks and at the end of study treatment. Thyroid function tests (free triiodothyronine $\left[\mathrm{T}_{3}\right]$, free thyroxine $\left[\mathrm{T}_{4}\right]$, and thyroid-stimulating hormone [TSH]) were performed at baseline. TSH was remeasured at weeks $2,4,8$, and 12, and every 8 weeks thereafter. Free $\mathrm{T}_{3} / \mathrm{T}_{4}$ was measured when clinically indicated. An independent third-party data monitoring committee monitored safety on a periodic basis and had the ability to recommend early termination of the trial.

Patient-reported outcomes were assessed using the FKSI$15,{ }^{30}$ FKSI-DRS, ${ }^{31}$ and European Quality of Life-5 Dimensions (EQ-5D). ${ }^{32}$ FKSI-15, a 15-item scale (score range, 0-60), measures symptoms and health-related quality of life (QoL) in patients with advanced RCC. ${ }^{30}$ The FKSI's 15 items include lack of energy, side effects, pain, losing weight, bone pain, fatigue, enjoying life, short of breath, worsened condition, appetite, coughing, bothered by fevers, ability to work, hematuria, and sleep. The FKSI-DRS consists of nine items (score range, 0-36) of the FKSI-15 that are specific to kidney cancer. ${ }^{31}$ Higher scores on the FKSI-15 and FKSI-DRS are reflective of a better health state. The EQ-5D is a brief generic questionnaire that measures general health status. ${ }^{32} \mathrm{FKSI}$ and EQ-5D were administered on day 1 before dosing and before any other clinical assessments, every 4 weeks while on study, and at end of study treatment/withdrawal and follow-up (28 days after the last dose).

\section{Statistical analyses}

The primary efficacy end point was IRC-assessed PFS from randomization to first documentation of objective 
tumor progression or death. Secondary end points included PFS assessed by the treating physicians; overall survival, defined as the time from the date of randomization to the date of death; IRC-assessed ORR, defined as the percentage of patients with confirmed (ie, persisted on repeat imaging for $\geq 4$ weeks) complete or partial response according to RECIST criteria; ${ }^{27}$ and duration of response, defined as the time from the first documentation of objective complete or partial tumor response and subsequently confirmed, to the first documentation of progressive disease or death.

Efficacy and patient-reported outcomes were analyzed in the intention-to-treat population (all randomized patients), and safety was analyzed in all patients who received at least one dose of study drug. Final PFS analysis was conducted when data were considered sufficiently mature (ie, after $\sim 130$ PFS events occurred or $\sim 60 \%$ of patients had a PFS event). The sample size of 200 patients was chosen based on requirements of the China Food and Drug Administration in order to support the submission of axitinib treatment for advanced RCC after failure of one prior systemic therapy. The trial was not powered to detect statistically significant differences between axitinib and sorafenib; all $P$-values are provided for reference only.

The Kaplan-Meier method was used to estimate median PFS and duration of response, and two-sided 95\% CI was calculated. PFS was compared between treatment arms using a stratified, one-sided, log-rank test. A Cox proportional hazard model was used to explore the potential influence of the baseline stratification factor and patient characteristics (ie, age, sex, and ethnic origin) on PFS. The estimated HR and two-sided 95\% CI were provided. Additionally, for each treatment arm, the median PFS and two-sided 95\% CI were provided for each level of the stratification factor or baseline characteristics. ORR for the two treatment arms was compared using a one-sided Pearson chi-square test for unstratified analyses and the Cochran-Mantel-Haenszel test for stratified analyses.

The mean (and 95\% CI) and median of the observed scores and the change from baseline were reported for FKSI-15, FKSI-DRS, and EQ-5D. A repeated-measures, mixed-effects model was used to compare the two treatment arms over the course of the study, and survival analysis methods were used to compare treatments with respect to time to deterioration, where deterioration was predefined as a composite end point of death, progression, or a minimum important decrease in FKSI-15 (5 points) ${ }^{30}$ or FKSI-DRS
(3 points), ${ }^{31}$ whichever occurred first. The data cutoff date for these analyses was October 31, 2011.

\section{Results}

\section{Patients and treatment}

Between August 2009 and March 2011, 204 previously treated patients with $\mathrm{mRCC}$ were enrolled. In all, 135 patients were randomly assigned to axitinib and 69 to sorafenib (Figure 1). Patient demographics and baseline characteristics were largely balanced between treatment arms (Table 1). The majority of Asian patients were Chinese; two patients assigned to axitinib were not Asian. Similar proportions of patients in the axitinib $(85.2 \%)$ and sorafenib (89.9\%) arms had previous surgery for nephrectomy. A greater proportion of patients assigned to axitinib compared with sorafenib had bone metastases at baseline (Table 1).

The proportion of patients with specific, present medical histories was similar between the treatment arms. In total, $82(60.7 \%)$ patients in the axitinib arm and $48(69.6 \%)$ patients in the sorafenib arm reported a present medical disease/syndrome. The most frequently reported present medical history was hypertension, reported by $30(22.2 \%)$ patients in the axitinib arm and $16(23.2 \%)$ patients in the sorafenib arm. A lower proportion of patients in the axitinib arm versus the sorafenib arm had hypothyroidism at baseline (six [4.4\%] versus nine [13.0\%] patients, respectively).

All patients received the assigned treatment. As of the data cutoff date, $85(63.0 \%)$ patients in the axitinib arm and $49(71.0 \%)$ patients in the sorafenib arm had discontinued study treatment. The most common reasons for treatment discontinuation for axitinib and sorafenib, respectively, were disease progression (39.3\% versus $50.7 \%)$ and death $(9.6 \%$ versus $7.2 \%$ ) (Table 1 ).

Median treatment duration was longer (9.1 versus 7.0 months) and median relative dose intensity (total dose administered/total dose assigned $\times 100)$ was greater $(106.4 \%$ versus $99.8 \%$ ) with axitinib and sorafenib, respectively. Axitinib dose was increased to $>5 \mathrm{mg}$ BID in $83(61.5 \%)$ patients. A smaller proportion of patients had dose reductions $(20.7 \%$ versus $31.9 \%)$, whereas a greater proportion had interruptions (66.7\% versus 56.5\%) with axitinib and sorafenib, respectively. AEs led to dose interruptions in $70(51.9 \%)$ and $24(34.8 \%)$ patients receiving axitinib and sorafenib, respectively.

During the study, patients were not offered axitinib or sorafenib as subsequent therapy following treatment 


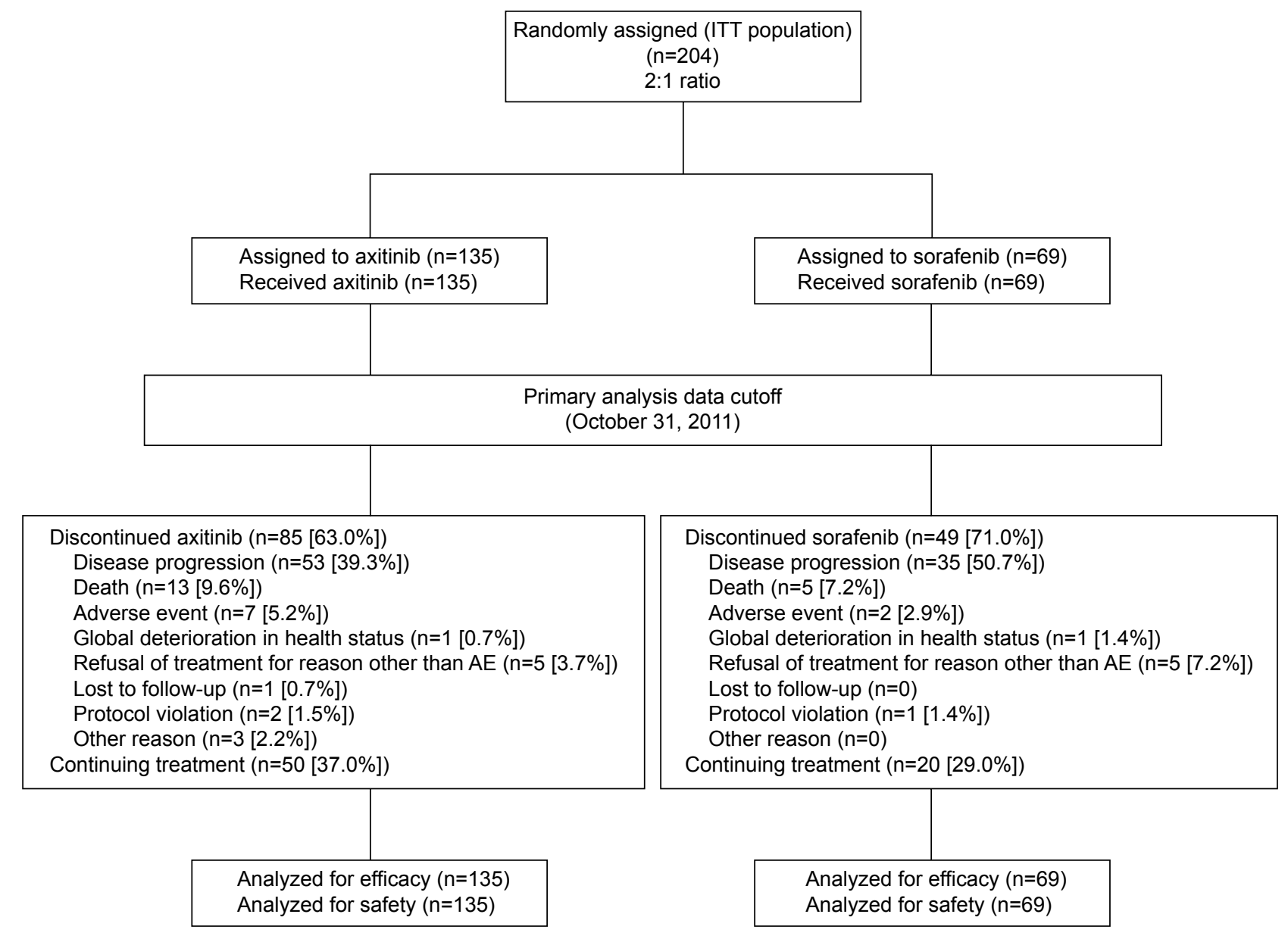

Figure I CONSORT diagram.

Abbreviations: AE, adverse event; ITT, intention-to-treat; CONSORT, Consolidated Standards of Reporting Trials.

discontinuation. Overall, eight (5.9\%) patients in the axitinib arm and nine (13.0\%) patients in the sorafenib arm received other follow-up systemic therapy.

\section{Efficacy}

Based on masked IRC review, 87 (64.4\%) patients in the axitinib arm and $46(66.7 \%)$ patients in the sorafenib arm had PFS events. The IRC-assessed median PFS (95\% CI) was 6.5 (4.7-9.1) months with axitinib compared with 4.8 (3.0-6.5) months with sorafenib (HR, 0.731; 95\% CI, $0.506-1.058 ; P=0.0531$ one-sided log-rank test stratified by ECOG PS and prior treatment; Figure 2A).

In patients previously treated with sunitinib, IRC-assessed median PFS (95\% CI) was 4.7 (4.5-6.5) months with axitinib compared with 2.8 (2.7-6.4) months with sorafenib (HR, 0.629; 95\% CI, 0.377-1.048; $P=0.0412$ one-sided log-rank test stratified by ECOG PS; Figure 2B). In patients previously treated with cytokines, IRC-assessed median PFS (95\% CI) was 10.1 (6.5-12.0) months with axitinib compared with 6.5 (4.8-12.0) months with sorafenib (HR, 0.860; 95\% CI, $0.502-1.475 ; P=0.2945$ one-sided log-rank test stratified by ECOG PS; Figure 2C).

Additional analyses of IRC-assessed PFS by patient characteristics and prognostic factors at baseline showed a generally consistent effect, numerically favoring axitinib in delaying PFS events relative to sorafenib (Figure 3). In the subgroup analyses, a treatment effect favoring axitinib was noted in females (HR, $0.441 ; 95 \% \mathrm{CI}, 0.226-0.860$ ), but not in males (HR, 0.945; 95\% CI, 0.615-1.453). However, when included in a multivariate Cox model adjusting for treatment and other baseline characteristics, sex was not an independent predictor of outcome.

Based on masked IRC assessment, confirmed ORR was $23.7 \%$ with axitinib versus $10.1 \%$ with sorafenib $(P=0.009$; Table 2). No patient had a complete response. In patients previously treated with a sunitinib-containing regimen, 
Table I Patient demographics and baseline characteristics

\begin{tabular}{|c|c|c|}
\hline & Axitinib $(n=135)$ & Sorafenib $(n=69)$ \\
\hline Age in years, median (range) & $56(|8-9|)$ & $56(22-82)$ \\
\hline \multicolumn{3}{|l|}{ Sex } \\
\hline Male & $94(69.6)$ & $49(71.0)$ \\
\hline Female & $4 \mathrm{I}(30.4)$ & $20(29.0)$ \\
\hline \multicolumn{3}{|l|}{ Race } \\
\hline White & $2(1.5)$ & 0 \\
\hline Asian & $133(98.5)$ & $69(100.0)$ \\
\hline Indian, subcontinent Asian & $\mathrm{I}(0.7)$ & 0 \\
\hline Southeast Asian & $4(3.0)$ & $2(2.9)$ \\
\hline Chinese & $128(94.8)$ & $67(97.1)$ \\
\hline \multicolumn{3}{|l|}{ ECOG PS } \\
\hline 0 & $82(60.7)$ & $42(60.9)$ \\
\hline I & $52(38.5)$ & $27(39.1)$ \\
\hline$>1$ & $\mathrm{I}(0.7)$ & 0 \\
\hline \multicolumn{3}{|l|}{ Prior systemic therapy ${ }^{\mathrm{b}}$} \\
\hline Sunitinib-containing & $67(49.6)$ & $34(49.3)$ \\
\hline Cytokine-containing & $68(50.4)$ & $35(50.7)$ \\
\hline \multicolumn{3}{|l|}{ Prior nephrectomy } \\
\hline No & $20(14.8)$ & $7(10.1)$ \\
\hline Yes & $115(85.2)$ & $62(89.9)$ \\
\hline \multicolumn{3}{|c|}{ MSKCC risk group [number risk factors] ${ }^{c}$} \\
\hline Favorable [0] & $47(34.8)$ & $23(33.3)$ \\
\hline Intermediate [I] & $54(40.0)$ & $30(43.5)$ \\
\hline Poor [2-3] & $34(25.2)$ & $16(23.2)$ \\
\hline \multicolumn{3}{|l|}{ Sites of metastasis } \\
\hline Lung & $112(83.0)$ & $54(78.3)$ \\
\hline Lymph node & $64(47.4)$ & $38(55.1)$ \\
\hline Bone & $52(38.5)$ & $15(21.7)$ \\
\hline Kidney & $34(25.2)$ & I5 (2I.7) \\
\hline Liver & $31(23.0)$ & $15(21.7)$ \\
\hline
\end{tabular}

Notes: Data are $\mathrm{n}(\%)$ unless noted. ${ }^{\mathrm{a} E C O G}$ PS from case report forms; last measure taken before dosing on or before randomization date. ${ }^{\text {PPrior }}$ therapy from interactive voice response system. 'MSKCC risk groups were classified using the following three risk factors: low serum hemoglobin (less than lower limit of normal), high corrected serum calcium ( $>10 \mathrm{mg} / \mathrm{dL}$ ), and ECOG PS (0 versus I). ${ }^{39}$

Abbreviations: ECOG PS, Eastern Cooperative Oncology Group performance status; MSKCC, Memorial Sloan Kettering Cancer Center.

IRC-assessed confirmed ORR was $14.9 \%$ with axitinib versus $5.9 \%$ with sorafenib $(P=0.0963)$. In patients previously treated with a cytokine-containing regimen, IRC-assessed confirmed ORR was $32.4 \%$ with axitinib versus $14.3 \%$ with sorafenib $(P=0.0240)$.

Based on masked IRC assessment, the median duration of response for the overall analysis in the axitinib arm was not estimable (95\% CI, 12.5 months - not estimable) compared with 8.7 months in the sorafenib arm (95\% CI, 4.1 months not estimable). Note that these CIs were not estimable as the majority of patients were still responding to treatment.

As of the data cutoff date, overall survival data were immature, with death reported in $44 \%$ of patients; patients are being followed for survival every 3 months following study treatment discontinuation for $\geq 3$ years after randomization of the last patient. The estimated survival probability (95\% CI) at 12 months was $67.0 \%(58.2 \%-74.4 \%)$ in the axitinib arm and $62.4 \%(49.2 \%-73.0 \%)$ in the sorafenib arm. The observed HR stratified by ECOG PS and prior treatment was 0.959 (95\% CI, 0.609-1.511) with one-sided $P=0.4289$.

\section{Safety}

The most frequently ( $\geq 30 \%$ ) reported all-grade, all-causality treatment-emergent AEs were hypertension (49.6\%), weight decrease $(37.0 \%)$, diarrhea (34.1\%), and palmar-plantar erythrodysesthesia (PPE; 31.9\%) with axitinib compared with PPE (56.5\%), hypertension (36.2\%), weight decrease (33.3\%), and diarrhea (30.4\%) with sorafenib (Table 3). The most frequently ( $\geq 5 \%$ ) reported grade $\geq 3$, all-causality AEs and laboratory abnormalities were hypertension, weight decrease, and proteinuria with axitinib compared with hypertension and PPE with sorafenib (Table 3). Ten (7.4\%) patients in the axitinib arm and four $(5.8 \%)$ patients in the sorafenib arm had grade 4 AEs (all causalities).

In the axitinib arm, 20 (14.8\%) patients died during the study (ie, grade 5 AEs) and 41 (30.4\%) died during follow-up compared with seven (10.1\%) and 21 (30.4\%) patients, respectively, in the sorafenib arm. The most frequently reported event leading to death during the study or follow-up was the disease under study. All-causality serious AEs were reported in 40 (29.6\%) patients receiving axitinib and 14 (20.3\%) patients receiving sorafenib. The most frequent serious $\mathrm{AE}$ was disease progression, experienced by $12(8.9 \%)$ patients in the axitinib arm and five (7.2\%) patients in the sorafenib arm.

Antihypertensive medications were used by $50.4 \%$ and $49.3 \%$ of patients in the axitinib and sorafenib arms, respectively, during the study. Before start of treatment, of patients treated with axitinib and sorafenib, respectively, three $(2.2 \%)$ versus four $(5.8 \%)$ patients were using thyroid medication, and after the first dose of study drug, 36 (26.7\%) versus eleven $(15.9 \%)$ patients started hypothyroidism medication or increased their dose of existing hypothyroidism medication. Among patients who had TSH $<5 \mu \mathrm{IU} / \mathrm{mL}$ before treatment, elevations of TSH to $\geq 10 \mu \mathrm{IU} / \mathrm{mL}$ occurred in 45 of 135 (34.4\%) patients receiving axitinib and eight of $69(11.9 \%)$ patients receiving sorafenib. Two patients (one in each treatment arm) were identified as having a total bilirubin $\geq 2 \times$ upper limit of normal and elevated alkaline phosphatase, which were likely caused by underlying RCC and metastases to the liver.

\section{Patient-reported outcomes}

Completion rates were $\geq 90 \%$ in both treatment arms for the FKSI-15, FKSI-DRS, and EQ-5D questionnaires during treatment, based on eligible patients. 


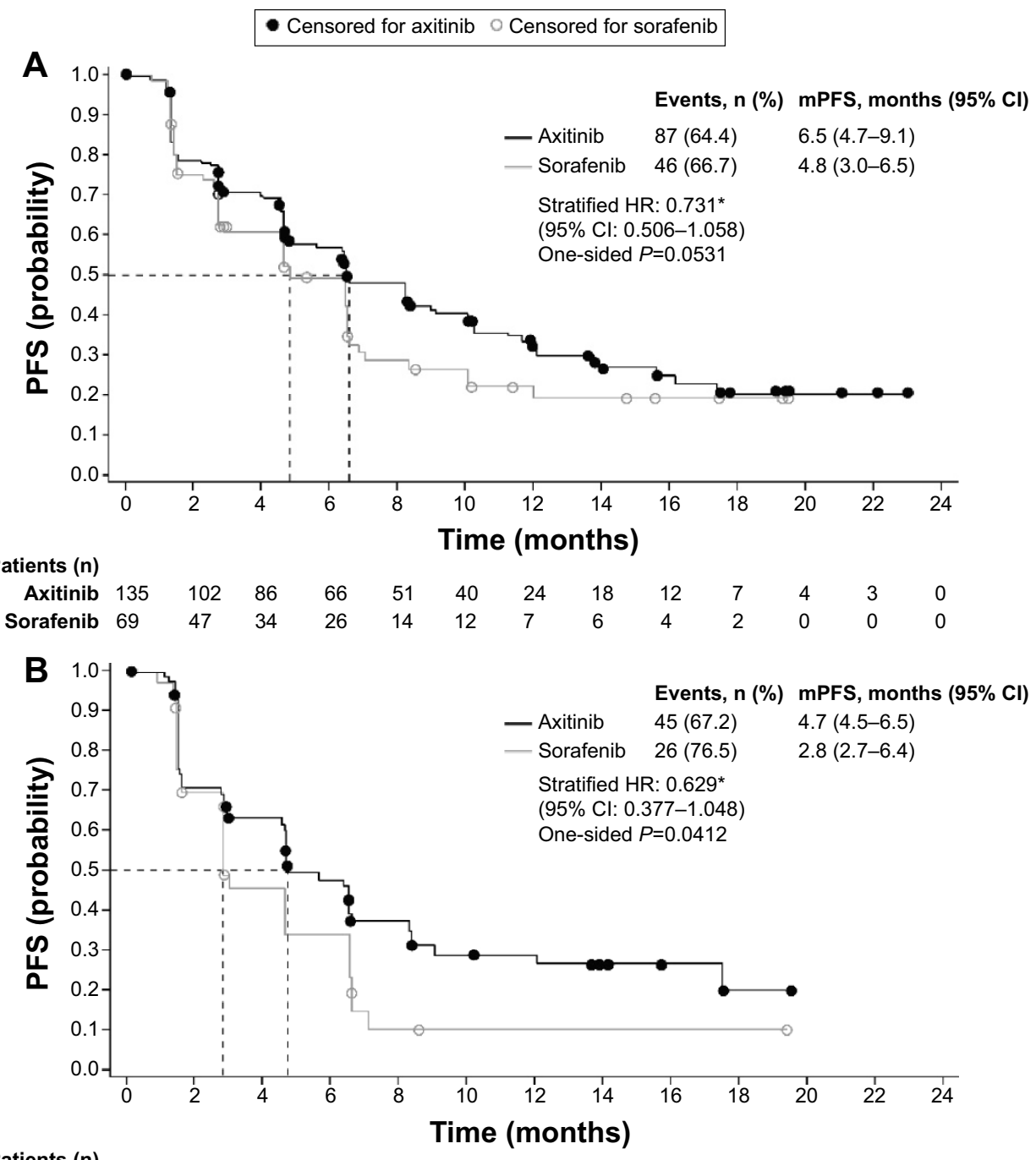

$\begin{array}{rlllllllllllll}\text { Patients (n) } & & & & & & & & & & \\ \text { Axitinib } & 67 & 45 & 38 & 27 & 18 & 13 & 9 & 7 & 4 & 1 & 0 & 0 & 0 \\ \text { Sorafenib } & 34 & 21 & 12 & 9 & 2 & 1 & 1 & 1 & 1 & 1 & 0 & 0 & 0\end{array}$

$\begin{array}{llllllllllllll}\text { Sorafenib } & 34 & 21 & 12 & 9 & 2 & 1 & 1 & 1 & 1 & 1 & 0 & 0 & 0\end{array}$

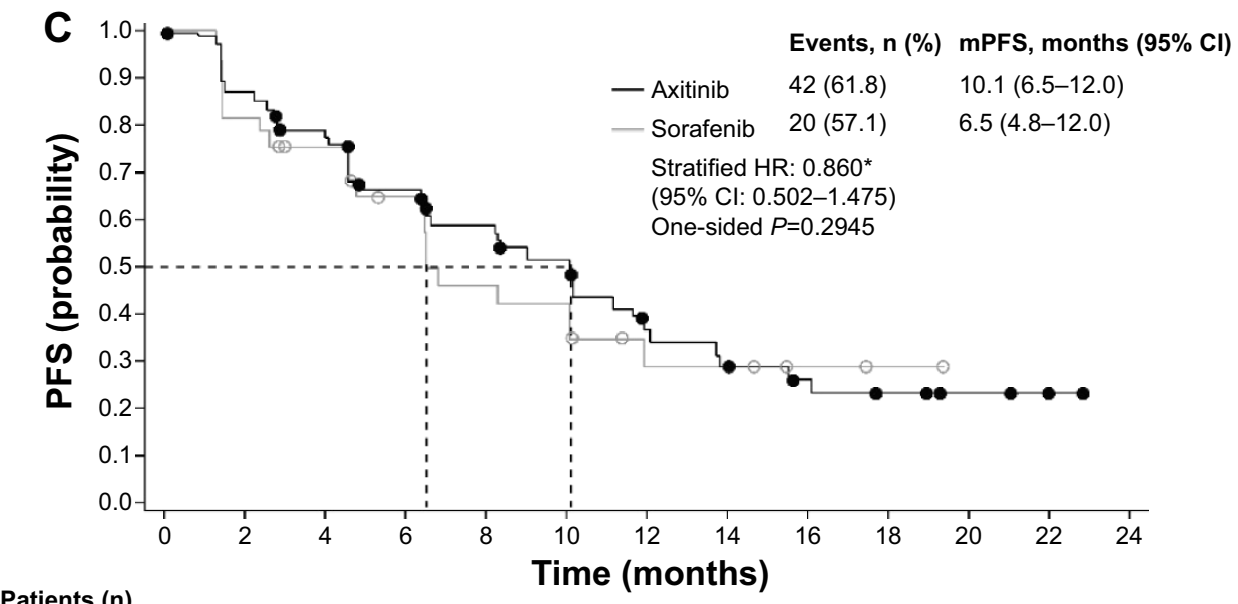

Patients ( $\mathrm{n}$ )

$\begin{array}{rlllllllllllll}\text { Axitinib } & 68 & 57 & 48 & 39 & 33 & 27 & 15 & 11 & 8 & 6 & 4 & 3 & 0 \\ \text { Sorafenib } & 35 & 26 & 22 & 17 & 12 & 11 & 6 & 5 & 3 & 1 & 0 & 0 & 0\end{array}$

Figure 2 Kaplan-Meier estimates of masked IRC-assessed PFS.

Notes: (A) intention-to-treat population, (B) patients previously treated with sunitinib, and (C) patients previously treated with cytokines. *Stratified by ECOG PS (and for prior therapy, in panel a only); assuming proportional hazards, $\mathrm{HR}<\mathrm{I}$ indicates a reduction in favor of axitinib and $\mathrm{HR}>\mathrm{I}$ indicates a reduction in favor of sorafenib. Abbreviations: $\mathrm{Cl}$, confidence interval; ECOG PS, Eastern Cooperative Oncology Group performance status; HR, hazard ratio; IRC, independent review committee; mPFS, median progression-free survival; PFS, progression-free survival. 


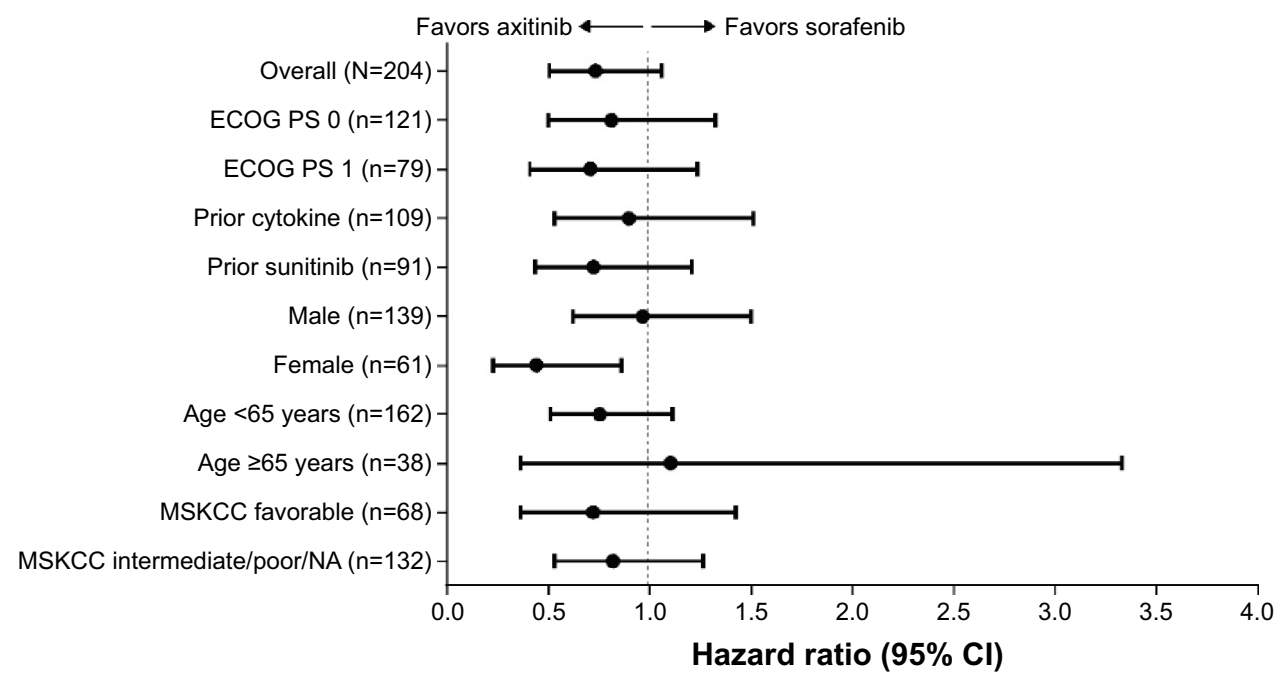

Figure 3 Subgroup analysis of masked IRC-assessed progression-free survival with respect to baseline characteristics and prognostic factors. Notes: Analysis excluded three patients with ECOG PS 0 who had not received first-line systemic therapy and one patient with ECOG PS 2 . ECOG PS and prior therapy obtained from case report forms.

Abbreviations: Cl, confidence interval; ECOG PS, Eastern Cooperative Oncology Group performance status; IRC, independent review committee; MSKCC, Memorial Sloan Kettering Cancer Center; NA, not applicable.

The mean FKSI-15 and FKSI-DRS values were similar for the two treatment arms at baseline and remained similar throughout the study until end of treatment. The FKSI-15 and FKSI-DRS scores worsened in both treatment arms at end of treatment, when most patients had disease progression.

The median time to deterioration based on FKSI-15 composite end point analysis was 4.6 months with axitinib versus 2.8 months with sorafenib (HR, 0.759; 95\% CI, 0.553-1.043; one-sided $P=0.0421$ ). The median time to deterioration based on FKSI-DRS composite end point analysis was 4.6 months with axitinib versus 3.0 months with sorafenib (HR, 0.830;

Table 2 Masked IRC-assessed best objective response rate

\begin{tabular}{|c|c|c|}
\hline & Axitinib $(n=135)$ & Sorafenib $(n=69)$ \\
\hline Overall ORR, \% (95\% Cl) & $23.7(|6.8-3| .8)$ & $10.1(4.2-19.8)$ \\
\hline Risk ratio $(95 \% \mathrm{Cl})$ & $2.339(1.094-5.002)$ & \\
\hline$P a$ & 0.009 & \\
\hline \multicolumn{3}{|c|}{ Best observed response, $\mathrm{n}(\%)^{\mathrm{b}}$} \\
\hline Complete response & 0 & 0 \\
\hline Partial response & $32(23.7)$ & $7(10.1)$ \\
\hline \multicolumn{3}{|l|}{ Stable disease } \\
\hline$\geq 20$ weeks & $34(25.2)$ & $19(27.5)$ \\
\hline$<20$ weeks & $30(22.2)$ & $15(21.7)$ \\
\hline Progressive disease & $24(17.8)$ & $16(23.2)$ \\
\hline Not assessed & $5(3.7)$ & $4(5.8)$ \\
\hline Indeterminate & $2(1.5)$ & $\mathrm{I}(\mathrm{l} .4)$ \\
\hline
\end{tabular}

Notes: ${ }^{a}$-value is from a one-sided Cochran-Mantel-Haenszel test stratified by Eastern Cooperative Oncology Group performance status and prior therapy. ${ }^{\text {bPer }}$ IRC assessment, eight patients in the axitinib arm and seven patients in the sorafenib arm did not have measureable disease at baseline.

Abbreviations: IRC, independent review committee; ORR, objective response rate; $\mathrm{Cl}$, confidence interval.
95\% CI, 0.600-1.149; one-sided $P=0.1277$ ). The overall comparison for axitinib and sorafenib, based on a repeatedmeasures mixed-effects model, was not statistically significant with respect to EQ-5D, FKSI-15, or FKSI-DRS.

\section{Discussion}

In this registrational study comparing two targeted agents in Asian patients with $\mathrm{mRCC}$, though not powered to detect statistically significant differences between the two treatments, treatment with axitinib resulted in a numerically longer PFS (6.5 versus 4.8 months; HR, 0.731; one-sided $P=0.0531)$ and higher ORR (23.7\% versus $10.1 \% ; P=0.009)$ compared with sorafenib, respectively. The outcome for sorafenib in the current study was similar to that in a global Phase III trial of sorafenib that supported its use as a secondline treatment for advanced RCC. ${ }^{19,33}$ The results among Asian patients treated with axitinib in the current study are also similar to those observed in the global Phase III AXIS trial $^{22}$ and the Asian subgroup of Japanese patients. ${ }^{23}$ The current study provides further evidence for the use of axitinib as a standard treatment in Asian patients with $\mathrm{mRCC}$ after the failure of a first-line systemic therapy.

Axitinib treatment resulted in numerically longer PFS and higher ORR in patients previously treated with cytokines than patients previously treated with sunitinib, which is consistent with the results from the global AXIS trial as well as the subgroup of patients from Japan. ${ }^{22,23}$ Cross-trial comparisons should be interpreted with caution because of potential methodologic and other differences. However, 
Table 3 Treatment-emergent, all-causality adverse events and laboratory abnormalities experienced by $\geq 15 \%$ of patients in either treatment group, by treatment (safety population)

\begin{tabular}{|c|c|c|c|c|}
\hline & \multicolumn{2}{|c|}{ Axitinib $(n=135)$} & \multicolumn{2}{|c|}{ Sorafenib $(n=69)$} \\
\hline & All grades & Grade $\geq 3$ & All grades & Grade $\geq 3$ \\
\hline \multicolumn{5}{|l|}{ Adverse event, $\mathrm{n}(\%)$} \\
\hline Hypertension & $67(49.6)$ & $26(19.3)$ & $25(36.2)$ & $6(8.7)$ \\
\hline Weight decrease & $50(37.0)$ & $7(5.2)$ & $23(33.3)$ & I (I.4) \\
\hline Diarrhea & $46(34.1)$ & $4(3.0)$ & $21(30.4)$ & I (I.4) \\
\hline PPE & $43(31.9)$ & $5(3.7)$ & $39(56.5)$ & $5(7.2)$ \\
\hline Decreased appetite & $40(29.6)$ & I (0.7) & $14(20.3)$ & $2(2.9)$ \\
\hline Hypothyroidism & $38(28.1)$ & I (0.7) & $16(23.2)$ & I (I.4) \\
\hline Fatigue & $36(26.7)$ & $4(3.0)$ & $16(23.2)$ & I (I.4) \\
\hline Proteinuria & $28(20.7)$ & $7(5.2)$ & $14(20.3)$ & $3(4.3)$ \\
\hline Dysphonia & $26(19.3)$ & I $(0.7)$ & $6(8.7)$ & 0 \\
\hline Cough & $22(16.3)$ & 0 & II (I5.9) & 0 \\
\hline Rash & $20(14.8)$ & 0 & $19(27.5)$ & I (I.4) \\
\hline Pyrexia & $9(6.7)$ & I (0.7) & II (I5.9) & I (I.4) \\
\hline Alopecia & $4(3.0)$ & 0 & I5 (2I.7) & I (I.4) \\
\hline \multicolumn{5}{|c|}{ Laboratory abnormality, n/N (\%) } \\
\hline \multicolumn{5}{|l|}{ Hematologic } \\
\hline Hemoglobin & $64 / 126(50.8)$ & $4 / 126(3.2)$ & $39 / 65(60.0)$ & $5 / 65(7.7)$ \\
\hline Lymphocytes & $60 / 129(46.5)$ & $9 / 129(7.0)$ & $37 / 66(56.1)$ & $5 / 66(7.6)$ \\
\hline Platelets & $39 / 123(31.7)$ & 0 & $17 / 62(27.4)$ & 0 \\
\hline White blood cells & $38 / 125(30.4)$ & 0 & I2/64 (I8.8) & 0 \\
\hline Neutrophils & $21 / 129(16.3)$ & 0 & $7 / 66(10.6)$ & 0 \\
\hline \multicolumn{5}{|l|}{ Biochemistry, n/N (\%) } \\
\hline Bicarbonate & $73 / I 24$ (58.9) & 0 & $4 I / 64(64 . I)$ & $\mathrm{I} / 64(\mathrm{I} .6)$ \\
\hline Hypocalcemia & $61 / 125(48.8)$ & 0 & $49 / 65(75.4)$ & $4 / 65(6.2)$ \\
\hline Alkaline phosphatase & $54 / 125(43.2)$ & $\mathrm{I} / \mathrm{I} 25(0.8)$ & 23/65 (35.4) & $3 / 65(4.6)$ \\
\hline Creatinine & $49 / 125$ (39.2) & $1 / 125(0.8)$ & 23/65 (35.4) & 0 \\
\hline AST & $44 / 125(35.2)$ & $2 / 125(1.6)$ & 22/65 (33.8) & 0 \\
\hline $\mathrm{ALT}$ & $43 / 125(34.4)$ & $2 / 125(1.6)$ & I8/65 (27.7) & 0 \\
\hline Amylase & $4 \mathrm{I} / \mathrm{I} 25$ (32.8) & $4 / 125(3.2)$ & $24 / 65(36.9)$ & $2 / 65(3.1)$ \\
\hline Lipase & $36 / 125(28.8)$ & $7 / 125(5.6)$ & $31 / 65(47.7)$ & $6 / 65(9.2)$ \\
\hline Hypoalbuminemia & $31 / 125(24.8)$ & $1 / / 25(0.8)$ & 22/65 (33.8) & $\mathrm{I} / 65(\mathrm{I} .5)$ \\
\hline Hyperglycemia & $27 / 125(21.6)$ & $\mathrm{I} / \mathrm{I} 25(0.8)$ & 10/65 (I5.4) & $3 / 65(4.6)$ \\
\hline Hypokalemia & $26 / 125(20.8)$ & $6 / 125(4.8)$ & I8/65 (27.7) & $5 / 65(7.7)$ \\
\hline Bilirubin & $25 / 125(20.0)$ & $\mathrm{I} / \mathrm{I} 25(0.8)$ & $6 / 65(9.2)$ & I/65 (I.5) \\
\hline Hypophosphatemia & $22 / 125(17.6)$ & $5 / 125(4.0)$ & $46 / 65(69.2)$ & $15 / 65(23.1)$ \\
\hline Hyponatremia & $15 / 125(12.0)$ & $6 / 125(4.8)$ & $12 / 65(18.5)$ & $3 / 65(4.6)$ \\
\hline
\end{tabular}

Notes: aDenominator for each laboratory abnormality differed depending on the availability of baseline and at least one postbaseline test result.

Abbreviations: PPE, palmar-plantar erythrodysesthesia; AST, aspartate aminotransferase; ALT, alanine aminotransferase.

among patients who failed a prior cytokine-containing regimen, the median PFS was 10.1 months with axitinib compared with 6.5 months with sorafenib reported in the current study (HR, 0.629; one-sided $P=0.0412$ ), and with the previously reported median PFS of 7.7 months with sunitinib ${ }^{7}$ in a similar Asian patient population. Because cytokines are still widely used as first-line therapy in Japan and China, the favorable results with axitinib support its use as second-line treatment for $\mathrm{mRCC}$ in these regions. ${ }^{17}$

The safety profile of axitinib and sorafenib in Asian patients was consistent with that seen in the global Phase III AXIS trial and with other VEGFR inhibitors..$^{9,22,33-36}$
Axitinib was generally well tolerated by Asian patients and the AE profile was similar to the general population in the AXIS study, with the exception of diarrhea, fatigue, and dysphonia, which were experienced less frequently by Asian patients, and for weight decrease, hypothyroidism, and hypertension, which were experienced more frequently by Asian patients. The reasons for those differences are not clear; however, it was noted in the clinical setting that diarrhea and fatigue were generally less frequently experienced by Asian patients than by patients from the United States or Europe who received sunitinib, sorafenib, or bevacizumab as first-line treatment. ${ }^{37}$ Overall, in the current study, AEs 
in Asian patients were manageable and reversible by dose modification, dose interruption, or symptomatic treatment.

Another goal for the management of $\mathrm{mRCC}$ is to maintain patients' functional outcomes and QoL. Axitinib prolonged PFS compared with sorafenib, and generally enabled patients to maintain QoL while on treatment (prior to disease progression). There was no difference between the two treatment arms in the FKSI-15 and FKSI-DRS observed mean scores at each cycle over time; however, as patients remained progression-free for a longer period of time while treated with axitinib compared with sorafenib, the study results suggest that axitinib-treated patients were able to maintain their levels of functioning and QoL for a longer period of time than patients treated with sorafenib. At the end of treatment, when most patients had discontinued treatment owing to disease progression, there was a worsening of FKSI-15 and FKSI-DRS scores in both treatment arms. Asian patients in the axitinib arm demonstrated a $17 \%-24 \%$ risk reduction in the time-to-deterioration composite end point of death, progression, and worsening of QoL, compared with sorafenib. Although not statistically significant, these results are consistent with risk reduction in time to deterioration observed in the general population in the AXIS study. ${ }^{22}$ The findings from this study combined with those previously reported for the AXIS trial ${ }^{22,38}$ demonstrate the importance of delaying disease progression and maintaining patient's QoL.

The results of this study should be considered with its limitations. The sample size in this study was small as it was chosen based on requirements of the China Food and Drug Administration. As a result, the trial was not powered to detect statistically significant differences between axitinib and sorafenib. The overall survival data are not mature at this time and will be reported separately.

\section{Conclusion}

Axitinib is clinically active and well tolerated in Asian patients with $\mathrm{mRCC}$, particularly Chinese patients, after the failure of one prior systemic therapy. This is consistent with the results in the overall population in the global AXIS trial. Second-line treatment with axitinib resulted in numerically longer PFS compared with sorafenib while generally enabling patients to maintain QoL. These results establish axitinib as a second-line treatment option for Asian patients with mRCC.

\section{Acknowledgments}

This study was sponsored by Pfizer Inc. Medical writing support was provided by Lilliam Poltorack, PharmD, and
Vardit Dror, PhD, of Engage Scientific Solutions and was funded by Pfizer Inc. The authors would like to thank Helen Bhattacharyya for her contribution to the analysis and interpretation of the PRO data and Lingjuan Pang of Pfizer Inc, for facilitating the authors' review of this manuscript.

\section{Disclosure}

JT, JT, and CC are full-time employees of Pfizer Inc. SK was a Pfizer employee at the time the study was conducted and owns shares in Pfizer and Mirna Therapeutics. SQ, FB, $\mathrm{JJ}, \mathrm{YC}, \mathrm{JG}, \mathrm{XR}, \mathrm{YH}$, and DY declare that they have no competing interests.

\section{References}

1. Wang YC, Wei LJ, Liu JT, Li SX, Wang QS. Comparison of cancer incidence between China and the USA. Cancer Biol Med. 2012;9(2): $128-132$.

2. International Agency for Research on Cancer. GLOBOCAN 2012 v1.0, Cancer Incidence and Mortality Worldwide: IARC CancerBase No 11. [Internet] Lyon, France: International Agency for Research on Cancer; 2013. Available from: http://globocan.iarc.fr. Accessed January 28, 2015.

3. National Comprehensive Cancer Network. NCCN Clinical Practice Guidelines in Oncology. Kidney Cancer. Version 1.2013. [Internet]; 2013. Available from: http://www.nccn.org/professionals/physician_gls/ PDF/kidney.pdf. Accessed January 28, 2015.

4. Tomita Y, Shinohara N, Yuasa T, et al. Overall survival and updated results from a phase II study of sunitinib in Japanese patients with metastatic renal cell carcinoma. Jpn J Clin Oncol. 2010;40(12): $1166-1172$.

5. Kim HS, Hong MH, Kim K, et al. Sunitinib for Asian patients with advanced renal cell carcinoma: a comparable efficacy with different toxicity profiles. Oncology. 2011;80(5-6):395-405.

6. Yoo C, Kim JE, Lee JL, et al. The efficacy and safety of sunitinib in Korean patients with advanced renal cell carcinoma: high incidence of toxicity leads to frequent dose reduction. Jpn J Clin Oncol. 2010;40(10): 980-985.

7. Uemura H, Shinohara N, Yuasa T, et al. A phase II study of sunitinib in Japanese patients with metastatic renal cell carcinoma: insights into the treatment, efficacy and safety. Jpn J Clin Oncol. 2010;40(3): 194-202.

8. Motzer RJ, Hutson TE, Cella D, et al. Pazopanib versus sunitinib in metastatic renal-cell carcinoma. N Engl J Med. 2013;369(8):722-731.

9. Sternberg CN, Davis ID, Mardiak J, et al. Pazopanib in locally advanced or metastatic renal cell carcinoma: results of a randomized phase III trial. J Clin Oncol. 2010;28(6):1061-1068.

10. Sternberg CN, Hawkins RE, Wagstaff J, et al. A randomised, doubleblind phase III study of pazopanib in patients with advanced and/or metastatic renal cell carcinoma: final overall survival results and safety update. Eur J Cancer. 2013;49(6):1287-1296.

11. Tanigawa G, Kawashima A, Yamaguchi S, et al. Clinical outcome and prognostic factors of sorafenib in Japanese patients with advanced renal cell carcinoma in general clinical practice. Jpn J Clin Oncol. 2011; 41(11):1265-1270.

12. Akaza H, Tsukamoto T, Murai M, Nakajima K, Naito S. Phase II study to investigate the efficacy, safety, and pharmacokinetics of sorafenib in Japanese patients with advanced renal cell carcinoma. Jpn J Clin Oncol. 2007;37(10):755-762.

13. Zhang H, Dong B, Lu JJ, et al. Efficacy of sorafenib on metastatic renal cell carcinoma in Asian patients: results from a multicenter study. BMC Cancer. 2009;9:249. 
14. Yang L, Shi L, Fu Q, et al. Efficacy and safety of sorafenib in advanced renal cell carcinoma patients: results from a long-term study. Oncol Lett. 2012;3(4):935-939.

15. Sun Y, Rha S, Lee SH, et al. Phase II study of the safety and efficacy of temsirolimus in East Asian patients with advanced renal cell carcinoma. Jpn J Clin Oncol. 2012;42(9):836-844.

16. Guo J, Huang Y, Zhang X, et al. Safety and efficacy of everolimus in Chinese patients with metastatic renal cell carcinoma resistant to vascular endothelial growth factor receptor-tyrosine kinase inhibitor therapy: an open-label phase 1b study. BMC Cancer. 2013;13:136.

17. Chiong E, Tay MH, Tan MH, et al. Management of kidney cancer in Asia: resource-stratified guidelines from the Asian Oncology Summit 2012. Lancet Oncol. 2012;13(11):e482-e491.

18. Wu B, Dong B, Xu Y, et al. Economic evaluation of first-line treatments for metastatic renal cell carcinoma: a cost-effectiveness analysis in a health resource-limited setting. PLoS One. 2012;7(3):e32530.

19. Beck J, Procopio G, Bajetta E, et al. Final results of the European Advanced Renal Cell Carcinoma Sorafenib (EU-ARCCS) expandedaccess study: a large open-label study in diverse community settings. Ann Oncol. 2011;22(8):1812-1823.

20. Hu-Lowe DD, Zou HY, Grazzini ML, et al. Nonclinical antiangiogenesis and antitumor activities of axitinib (AG-013736), an oral, potent, and selective inhibitor of vascular endothelial growth factor receptor tyrosine kinases 1, 2, 3. Clin Cancer Res. 2008;14(22):7272-7283.

21. Inlyta ${ }^{\circledR}$ (axitinib) [prescribing information]. New York, NY: Pfizer Inc; 2012.

22. Rini BI, Escudier B, Tomczak P, et al. Comparative effectiveness of axitinib versus sorafenib in advanced renal cell carcinoma (AXIS): a randomised phase 3 trial. Lancet. 2011;378(9807):1931-1939.

23. Ueda T, Uemura H, Tomita Y, et al. Efficacy and safety of axitinib versus sorafenib in metastatic renal cell carcinoma: subgroup analysis of Japanese patients from the global randomized phase 3 AXIS trial. Jpn J Clin Oncol. 2013;43(6):616-628.

24. Pithavala YK, Tortorici M, Toh M, et al. Effect of rifampin on the pharmacokinetics of axitinib (AG-013736) in Japanese and Caucasian healthy volunteers. Cancer Chemother Pharmacol. 2010;65(3):563-570.

25. Mukohara T, Nakajima H, Mukai H, et al. Effect of axitinib (AG-013736) on fatigue, thyroid-stimulating hormone, and biomarkers: a phase I study in Japanese patients. Cancer Sci. 2010;101(4):963-968.

26. Chen Y, Jiang J, Zhang J, et al. A phase I study to evaluate the pharmacokinetics of axitinib (AG-13736) in healthy Chinese volunteers. Int J Clin Pharmacol Ther. 2011;49(11):679-687.

27. Therasse P, Arbuck SG, Eisenhauer EA, et al. New guidelines to evaluate the response to treatment in solid tumors. J Natl Cancer Inst. 2000 92(3):205-216.
28. Hutson TE, Lesovoy V, Al-Shukri S, et al. Axitinib versus sorafenib as first-line therapy in patients with metastatic renal-cell carcinoma: a randomised open-label phase 3 trial. Lancet Oncol. 2013;14(13): 1287-1294.

29. Motzer RJ, Escudier B, Tomczak P, et al. Axitinib versus sorafenib as second-line treatment for advanced renal cell carcinoma: overall survival analysis and updated results from a randomised phase 3 trial. Lancet Oncol. 2013;14(6):552-562.

30. Cella D, Yount S, Du H, et al. Development and validation of the Functional Assessment of Cancer Therapy-Kidney Symptom Index (FKSI). J Support Oncol. 2006;4(4):191-199.

31. Cella D, Yount S, Brucker PS, et al. Development and validation of a scale to measure disease-related symptoms of kidney cancer. Value Health. 2007;10(4):285-293.

32. Kind P. The EuroQol instrument: an index of health-related quality of life. In: Spilker B, editor. Quality of Life and Pharmacoeconomics in Clinical Trials. 2nd ed. Philadelphia, PA: Lippicott-Raven; 1996: 191-201.

33. Escudier B, Eisen T, Stadler WM, et al. Sorafenib in advanced clear-cell renal-cell carcinoma. $N$ Engl J Med. 2007;356(2):125-134.

34. Motzer RJ, Hutson TE, Reeves J, et al. Randomized, open-label, phase III trial of pazopanib versus sunitinib in first-line treatment of patients with metastatic renal cell carcinoma (MRCC): results of the COMPARZ trial [abstract]. Ann Oncol. 2012;23(Suppl 9):LBA8_PR.

35. Motzer RJ, Hutson TE, Tomczak P, et al. Sunitinib versus interferon alfa in metastatic renal-cell carcinoma. $N$ Engl J Med. 2007;356(2): $115-124$.

36. Motzer RJ, Nosov D, Eisen T, et al. Tivozanib versus sorafenib as initial targeted therapy for patients with advanced renal cell carcinoma: results from a phase III randomized, open-label, multicenter trial [abstract]. J Clin Oncol. 2012;30(15 Suppl):4501.

37. Oh WK, McDermott D, Porta C, et al. Angiogenesis inhibitor therapies for advanced renal cell carcinoma: toxicity and treatment patterns in clinical practice from a global medical chart review. Int J Oncol. 2014; 44(1):5-16.

38. Cella D, Escudier B, Rini B, et al. Patient-reported outcomes for axitinib vs sorafenib in metastatic renal cell carcinoma: phase III (AXIS) trial. Br J Cancer. 2013;108(8):1571-1578.

39. Motzer RJ, Bacik J, Schwartz LH, et al. Prognostic factors for survival in previously treated patients with metastatic renal cell carcinoma. J Clin Oncol. 2004;22(3):454-463.
OncoTargets and Therapy

\section{Publish your work in this journal}

OncoTargets and Therapy is an international, peer-reviewed, open access journal focusing on the pathological basis of all cancers, potential targets for therapy and treatment protocols employed to improve the management of cancer patients. The journal also focuses on the impact of management programs and new therapeutic agents and protocols on

\section{Dovepress}

patient perspectives such as quality of life, adherence and satisfaction. The manuscript management system is completely online and includes a very quick and fair peer-review system, which is all easy to use. Visit http://www.dovepress.com/testimonials.php to read real quotes from published authors. 